\title{
Tilt-modulus enhancement of the vortex lattice in the layered superconductor $2 \mathrm{H}$-NbSe 2
}

\author{
P. Koorevaar, J. Aarts, P. Berghuis, and P. H. Kes \\ Kamerlingh Onnes Laboratory, Leiden University, P.O. Box 9506, 2300 RA Leiden, The Netherlands
}

(Received 11 April 1990)

\begin{abstract}
The field dependence of the pinning force has been studied in thin single crystals of the layered superconductor $2 \mathrm{H}-\mathrm{NbSe}_{2}$ in fields directed perpendicular to the layers. At high fields a peak effect is observed which sets in at about $B_{\text {co }} \approx 0.8 B_{c 2}$. Below this field the pinning force agrees well with the theory of two-dimensional collective pinning. The onset of the peak is triggered by the transition to three-dimensional flux-line lattice (FLL) disorder at the field $B_{\text {co. }}$. Comparison of the crossover field with the criterion set by the collective-pinning theory reveals that the tilt modulus of the FLL in a layered superconductor is considerably reduced. The reduction factor corresponds very well to recent theoretical predictions. These results are of importance for the prediction of depinning and flux-line lattice melting in all kinds of anisotropic superconductors.
\end{abstract}

The discovery of the high-temperature superconductors (HTS) has greatly stimulated the study of flux-line lattice (FLL) properties. One of the many interesting predictions ${ }^{1}$ is the dependence of the nonlocal tilt modulus of the FLL $c_{44}$ on the anisotropy parameter $\Gamma$ of layered superconductors. Here $\Gamma$ is the ratio between the effective electron masses perpendicular $\left(m_{z}\right)$ and parallel $(m)$ to the layers, $\Gamma=m_{z} / m$. In essence, with increasing $\Gamma$, the flux lines become more disklike ${ }^{2}$ leading to a reduction of $c_{44}$. FLL entanglement, ${ }^{3}$ FLL melting, 1,4 "giant" flux creep, depinning temperature, ${ }^{6}$ and vortex glass-to-liquid phase transition ${ }^{7.8}$ are among the new issues that are related to the peculiar characteristics of the extremely anisotropic HTS.

It is of great importance for the study of all these phenomena to test the predictions given by Houghton, Pelcovits, and Subdø. ' Information on the actual value of $c_{44}$ can be obtained from the field and temperature at which the crossover takes place from a two-dimensionally (2D) disordered (straight) FLL to a three-dimensionally disordered spaghetti state. ${ }^{9,10}$ Such a transition reveals itself by a sudden increase of the pinning force $F_{p}$ (peak effect) at the crossover field $B_{\text {co }}=b_{\text {co }} B_{c 2}$. The most convenient experimental conditions for observing the crossover are obtained for weak-pinning thin-film superconductors, so that the behavior for $B<B_{\text {co }}$ is well described by the $2 \mathrm{D}$ collective-pinning (2DCP) theory ${ }^{11,12}$ and $B_{\mathrm{co}} \approx 0.8 B_{c 2}$ is within experimental range. The HTS themselves are not well suited for these investigations because of strong fluxcreep effects and therefore we concentrated our research on a low-temperature layered superconductor, $2 \mathrm{H}-\mathrm{NbSe}_{2}$, which is an anisotropic type-II superconductor with $T_{c} \approx 7.3 \mathrm{~K}$. The structure has been well characterized ${ }^{13}$ and consists of $\mathrm{Nb}$ planes sandwiched between two Se planes. The anisotropy of the superconducting parameters has been the subject of several studies. ${ }^{14,15}$ The Ginzburg-Landau (GL) coherence length perpendicular to the layers is much larger than the distance between two superconducting $\mathrm{Nb}$ layers. Therefore the 3D GL theory for anisotropic superconductors describes the phenomena reasonably well.

For randomly distributed pinning centers (pins) no long-range order (LRO) is present in the FLL. " Shortrange order remains in elastically independent correlated regions with volume $V_{c}=R_{c}^{2} L_{c}$, where $R_{c}$ and $L_{c}$ are the transverse and longitudinal correlation lengths, respectively. The strength of the pinning is given by the parameter $W$ which depends on the concentration of the pins and their elementary interaction with the FLL. The pinningforce density $F_{p}$ and the critical-current density $J_{c}$ follow from

$$
F_{p}=B J_{c}=\left(W / V_{c}\right)^{1 / 2} .
$$

If elastic deformations are predominant the correlation lengths can be derived from the balance of elastic energy and pin energy. This situation only occurs if the disorder in the direction of the field can be neglected. 9,12 In that case $L_{c}=d$ (this is the 2DCP case) and $R_{c}$ is given by

$$
R_{c}=r_{f} c_{66}\left\{8 \pi d /\left[W \ln \left(w / R_{c}\right)\right]\right\}^{1 / 2}
$$

Here $r_{f}$ is the range of the elementary pinning force, $w$ the width, and $d$ the thickness of a thin film with the field applied perpendicular to the film, and $c_{66}$ is the shear modulus of the FLL, which according to Brandt, is given by

$$
c_{66}=\left(B_{c}^{2} / 4 \mu_{0}\right) b(1-0.29 b)(1-b)^{2}
$$

for large $\kappa$ (GL parameter) superconductors. $B_{c}$ is the thermodynamic critical field and $b=B / B_{c 2}$. For fields $b>0.2$ one can put $r_{f} \approx a_{0} / 2$. The situation of 2DCP is characterized by $F_{p} \propto B^{1 / 2}$ for low fields. For thin films a small peak effect is observed close to $B_{c 2}$ as soon as plastic deformations become predominant. For thick films a dimensional crossover (DCO) to 3D disorder results in a steep increase of $F_{p}$ caused by a highly defective FLL.

It has been determined empirically that the DCO sets in when

$$
L_{c}=\left(c_{44} / c_{66}\right)^{1 / 2} R_{c}=d / 2,
$$

where $c_{44}$ is the tilt modulus of the FLL. Brandt ${ }^{16}$ demonstrated that $c_{44}$ depends on the wave vector $\mathbf{k}=\left(k_{\perp}=\left(k_{x}^{2}+k_{y}^{2}\right)^{1 / 2}, k_{z}\right)$ of the deformation field. For 
isotropic superconductors

$$
c_{44}\left(k_{\perp}, k_{z}\right) \approx\left(B^{2} / \mu_{0}\right) k_{h}^{2} /\left(k_{\perp}^{2}+k_{z}^{2}+k_{h}^{2}\right)
$$

with $k_{h}^{2}=0.86(1-b) / \lambda^{2}$ and $\lambda$ the penetration depth. It has been explained in Ref. 17 that at the DCO $k_{\perp} \ll k_{B}$ and $k_{z} \approx \xi^{-1}$, so that $k_{z} \gg k_{B}$ and $k_{z} \gg k_{h}$. Here $k_{B}$ is the radius of the Brillouin zone in the circular cell approximation $k_{B}^{2}=8 \pi / a_{0}^{2} \sqrt{3}$ and $a_{0}=(2 / \sqrt{3})^{1 / 2}\left(\phi_{0} / B\right)^{1 / 2}$ is the FLL parameter. The condition $k_{z} \approx \xi^{-1}$ reflects that the core-size of a screw dislocation is of the order $\xi$, and that, as soon as $L_{c}$ related to the smallest possible wavelength fulfills the DCO criterion, screw dislocations are spontaneously created, probably at the surface. Substituting $c_{44}$ and $c_{66}$ in (3) yields for an isotropic superconductor that $\left(L_{c} / R_{c}\right)_{\mathrm{co}} \approx 3\left[b_{\mathrm{co}} /\left(1-b_{\mathrm{co}}\right)\right]^{1 / 2}$ at the DCO.

For anisotropic superconductors $c_{44}$ is much softer and is given by ${ }^{1}$

$$
\begin{aligned}
c_{44}\left(k_{\perp}, k_{z}\right)= & c_{44}(0) \frac{m}{m_{z}} \\
& \times\left(\frac{k_{h}^{2}}{k_{\perp}^{2}+\left(m / m_{z}\right)\left(k_{h}^{2}+k_{z}^{2}\right)}+\frac{1-b}{2 b \kappa^{2}}\right),
\end{aligned}
$$

with $c_{44}(0)=B^{2} / \mu_{0}$. The ratio $\mathrm{m} / \mathrm{m}_{z}$ is experimentally obtained from the upper-critical-field slopes in both orientations using $m / m_{z}=\left(S_{\perp} / S_{\|}\right)^{2}$ with $S_{\|, \perp}=-d B_{c 2 \|, \perp} /$ $\left.d T\right|_{T_{c}}$. As we will see below for the $\mathrm{NbSe}_{2}$ samples under investigation, $R_{c}\left(b_{\mathrm{co}}\right) \gg a_{0}$ at the $\mathrm{DCO}$, so that the most important deformations have wave vectors $k_{\perp}^{2} \ll k_{B}^{2}$. For $k_{z}$ we should now substitute $\xi_{z}^{-1}$, where $\xi_{z}$ is the GL coherence length perpendicular to the Nb layers. Substitution in (4) and (3) yields both $c_{44}$ and $L_{c}$ at the DCO,

$$
c_{44, \mathrm{co}}=\left(B_{c}^{2} / \mu_{0}\right)\left(\mathrm{m} / m_{z}\right)\left(1+1.72 b_{\mathrm{co}}\right) b_{\mathrm{co}}\left(1-b_{\mathrm{co}}\right)
$$

and

$$
\begin{aligned}
\frac{L_{c, \mathrm{co}}}{R_{c}\left(b_{\mathrm{co}}\right)} & =2\left(\frac{m}{m_{z}}\right)^{1 / 2}\left(\frac{1+1.72 b_{\mathrm{co}}}{\left(1-0.29 b_{\mathrm{co}}\right)\left(1-b_{\mathrm{co}}\right)}\right)^{1 / 2} \\
& \approx 3.93\left(\frac{m}{m_{z}}\right)^{1 / 2}\left(\frac{b_{\mathrm{co}}}{1-b_{\mathrm{co}}}\right)^{1 / 2}
\end{aligned}
$$

For $\boldsymbol{R}_{c}\left(b_{\mathrm{co}}\right)$ the value following from (2) should be used. The approximation of the prefactor 3.93 is better than $1 \%$ for $b>0.7$. The crossover field follows from $L_{c}=d / 2$.

In order to enable the observation of $2 \mathrm{DCP}$ we repeatedly cleaved as-grown $2 \mathrm{H}-\mathrm{NbSe}_{2}$ single crystals to obtain the smallest possible thickness. A well-known procedure was used of sandwiching the crystals between tape stuck onto two object slides. Taking the slides apart cleaves the crystals without causing significant surface damage as far as light-microscope inspection can show. The tape was removed by solving it in toluene for about $12 \mathrm{~h}$. This provided us with crystals ranging in thickness between 2 and 50 $\mu \mathrm{m}$. Bar-shaped samples were prepared by cutting the thinnest crystals to a typical size of $1 \times 5 \mathrm{~mm}$. The measurements discussed in this paper were carried out on a sample with $d=2 \mu \mathrm{m}$ (sample I) and on one with $d=15.5$ $\mu \mathrm{m}$ (sample II). Contacts for four-probe resistance measurements were made by silver paint.
The $T_{c}$ 's determined from the midpoints of the $R$ vs $T$ curves of samples I and II are 7.26 and $7.35 \mathrm{~K}$, respectively. The transition widths $\Delta T_{c}$ of $150 \mathrm{mK}$ were defined by the intercepts between the $10 \%$ and $90 \%$ normal-state resistances. Typical values of $T_{c}=7.18-7.39 \mathrm{~K}$ reported by Toyota et al. ${ }^{14}$ are close to ours. The residual resistance ratio of our samples is $R(T=293 \mathrm{~K}) / R(T=7.5$ $\mathrm{K})=34$, the room-temperature resistivity is $110 \times 10^{-8}$ $\Omega \mathrm{m}$.

Upper critical fields were determined from $R$ vs $B$ transitions at constant temperatures. The current densities were kept below $3 \times 10^{4} \mathrm{~A} / \mathrm{m}^{2}$ in order to suppress fluxflow effects. The characteristics of the transitions in increasing field can be described as a distinct onset, followed by a linear increase, changing to a gradual approach of the normal-state resistance $R_{n} . B_{c 2}$ is defined by extrapolating the linear part to $R=0$ and the transition width $\Delta B$ by the intercept between $R=0$ and $R=R_{n}$. For instance, we obtained for sample II at $5.0 \mathrm{~K} \quad B_{c 2}=1.51 T$ and $\Delta B=0.26 T$. It shows that the transitions are relatively broad, indicating the presence of inhomogeneities, probably caused by the splicing procedure. Comparison with Toyota's data ${ }^{14}$ on a single crystal with $T_{c}=7.29 \mathrm{~K}$ supports this conclusion. Our $B_{c 2}$ values coincide with his results, but our $\Delta B$ values are significantly larger.

The $J_{c}$ values were determined both from the measured IV curves and from the registration of $J_{c}(B)$ at a constant-voltage criterion of $2 \mu \mathrm{V} / \mathrm{cm}$. The characteristic features discussed below do not strongly depend on the criterion as long as it is within a factor of 3 of $2 \mu \mathrm{V} / \mathrm{cm}$. Typical results for sample II are shown in Fig. 1 for $T=1.8$ and $4.2 \mathrm{~K}$ in a plot of $F_{p}=J_{c} B$ vs $b=B / B_{c 2}$. We have used the $B_{c 2}$ values as obtained from the $R(B)$ transitions at the same temperature. Both the characteristic features of 2DCP and of a DCO are clearly observed: 9,12 a smoothly increasing $F_{p}$ roughly $\propto b^{1 / 2}$ up to $b \approx 0.7-$ 0.8 , a rapid increase (peak effect) up to a well-defined maximum followed by a sharp and almost linear decrease to zero. The size of the peak decreases with increasing temperature.

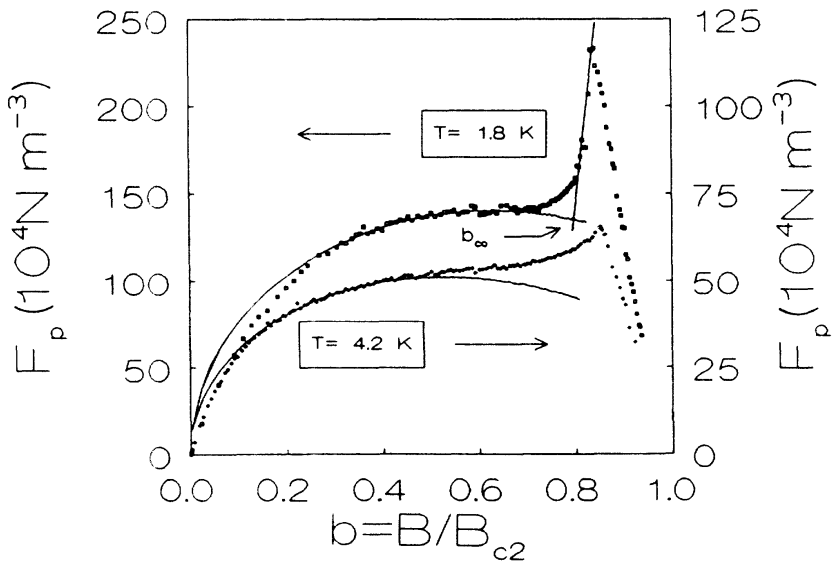

FIG. 1. Pinning force density vs $b$ at $t=0.245(T=1.8 \mathrm{~K})$ and $t=0.571(T=4.2 \mathrm{~K})$ for sample II. The solid lines represent the ideal 2DCP behavior. For $T=1.8 \mathrm{~K}$ the construction procedure for $b_{\text {co }}$ is shown. 
The low-field $F_{p}(b)$ behavior is typical for 2DCP and this regime provides the information needed to compute $\boldsymbol{R}_{c}$ and $\boldsymbol{L}_{c}$. Using Eqs. (1) and (2) with $\boldsymbol{r}_{f}=a_{0} / 2$, valid for $b>0.2$, and $V_{c}=R_{c}^{2} d$ yields $R_{c} / a_{0}$ and $W$ as a function of $b$. For $R_{c} / a_{0}(b)$ we observed the same domeshaped behavior in the $2 \mathrm{D}$ regime like for amorphous $\mathrm{Nb}_{3} \mathrm{Ge},{ }^{9}$ but the maximum values for $\boldsymbol{R}_{c} / a_{0}$, occurring when $b \approx 0.35$, are about 120 for sample $I$ and 240 for sample II, and are therefore much larger than in $\mathrm{Nb}_{3} \mathrm{Ge}$. The large $R_{c} / a_{0}$ values in the $2 \mathrm{D}$ regime indicate very large correlated volumes and thus very weak pinning, in good agreement with the scanning-tunneling-microscopy observations by Hess et al. ${ }^{18}$ which showed a very wellordered FLL. At the onset field of the peak effect $R_{c} / a_{0}$ has decreased to $\approx 100$ for sample II and $\approx 30$ for sample I.

For sample II we plotted the results for $W(b)$ as $W / b(1-b)^{2}$ vs $b$ in Fig. 2 for $T=1.8$ and $4.2 \mathrm{~K}$. One should realize that the computed data points are also plotted in the high-field regime where the 2DCP theory is not valid. The computed behavior of $W(b)$ is typical ${ }^{17,19,20}$ for pinning by defects which both have a $\delta T_{c^{-}}$and a $\delta \kappa-$ pinning character, e.g., small, flat precipitates parallel to the layers with different $T_{c}$. For such pins $W$ can be expressed as

$$
W=\left[C_{1}(t)+C_{2}(t) b\right] b(1-b)^{2},
$$

with $^{12,19-22}$

$$
C_{1}(t) \approx C B_{c 2}(t) B_{c}(t)^{2}\left(1+A t^{2}\right)^{2},
$$

and $C_{2}(t) \propto C_{1}(t) . C$ is a constant depending on the concentration of pins, and $|A|$ a constant of order 1. Figure 2 shows that the behavior according to (6) is observed for

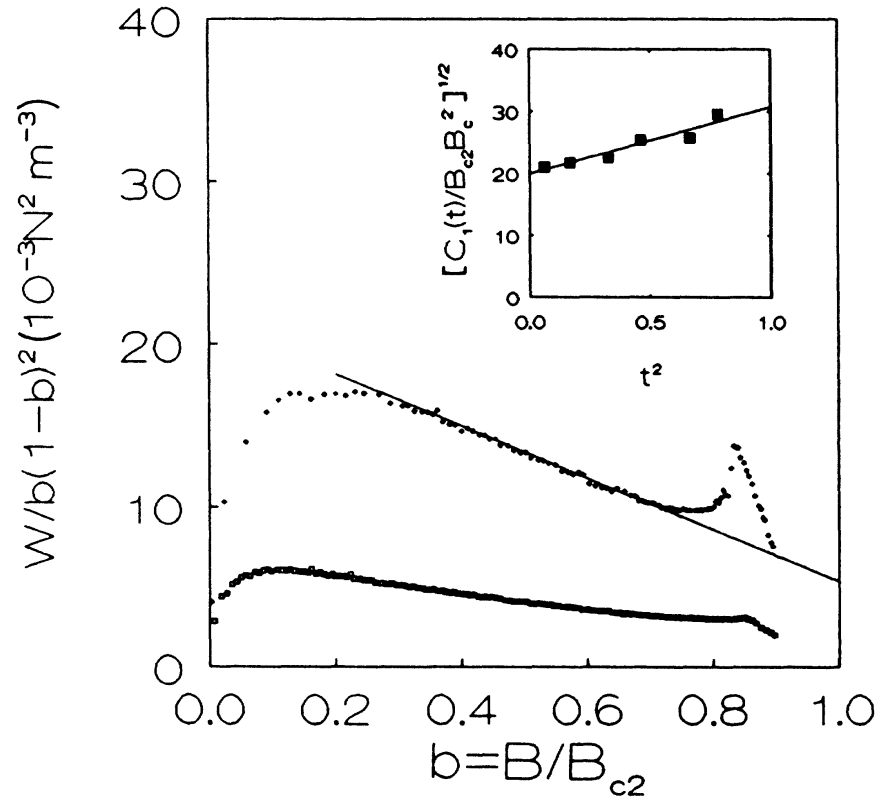

FIG. 2. $W / b(1-b)^{2}$ vs $b$ at $T=1.8 \mathrm{~K}$ (upper curve) and $T=4.2 \mathrm{~K}$ (lower curve) for sample II. The solid line represents the best fit of Eq. (6) to the data in the 2DCP regime. In the inset $\left(C_{1} / B_{c_{2} 2} B_{c}^{2}\right)^{1 / 2}$ is plotted vs $t^{2}$ for the same sample. The solid line represents Eq. (7) with $A=0.55$. $0.2<b<0.7$. Below $b=0.2$ deviations arise because $r_{f}$ $\approx \xi$ instead of $r_{f}=a_{0} / 2$ has to be used. ${ }^{23}$ The inset of Fig. 2 displays a plot of $\left(C_{1} / B_{c 2} B_{c}^{2}\right)^{1 / 2}$ vs $t^{2}$ for sample II and it is seen that the result is in accordance with Eq. (7) with $A=0.5 \pm 0.1$. The ratio $C_{1} / C_{2}$ has an average value -1.3 and is constant within $25 \%$. The above observations indicate that the pinning in $2 \mathrm{H}-\mathrm{NbSe}_{2}$ is indeed caused by small, flat precipitates.

We finally need to discuss the nature of the peak effect. In the original data (Fig. 1) the onset field for the peak is not sharply defined. Therefore we compute $F_{p}(b)$ from Eqs. (1), (2), and (6), using fitted values for $C_{1}(t)$ and $C_{2}(t)$. The result is given by the solid lines in Fig. 1. Above $b \approx 0.7, F_{p}$ starts to deviate from this ideal $2 \mathrm{DCP}$ behavior. We now define the onset field of the peak effect in a manner displayed in Fig. 1 (upper curve). The reason for the gradual transition will be discussed below. The peak effect may indicate the development of plastic deformations in the FLL in the form of edge dislocations. ${ }^{24}$ Such defects locally reduce $c_{66}$ so that the FL's can better adjust to the random pin distribution causing an increase of $F_{p}$. It has, however, been shown ${ }^{24}$ that plastic disorder develops when $R_{c} \leq 17 a_{0}$. Since in our samples $R_{c} / a_{0}$ at the peak onset is considerably larger, see Table I, we conclude that a crossover from two- to three-dimensional FLL disorder is a more likely explanation. Assuming then that the peak effect is caused by a DCO, we computed $L_{c}$ at $b_{\text {co }}$ from Eq. (5) substituting $m_{z} / m \approx 9$ as deduced from the $\boldsymbol{H}_{c 211} / \boldsymbol{H}_{c 2 \perp}$ data in Ref. 14. In Table I the values of both $b_{\text {co }}$ and the resulting $L_{c, \mathrm{co}} / d$ are listed. The error margins, given between parentheses, are related to the uncertainty in $b_{\text {co }}$. The temperature dependence of $L_{c, c o} / d$ is similar to that observed for thick amorphous $\mathrm{Nb}_{3} \mathrm{Ge}$ films in which a DCO has been unambiguously demonstrated. ${ }^{9}$ The agreement with the criterion $L_{c, \text { co }}=d / 2$ is better for lower temperatures in thicker films. From this we conclude that the peak effect in our $\mathbf{N b S e}_{2}$ samples is caused by a DCO. We note that it is crucial to take into account the anisotropy, for $L_{c} / d$ would be larger by a factor of 3 if we had used $m / m_{z}=1$.

The only question that remains is the different character of the peak observed here and for the DCO in amorphous $\mathrm{Nb}_{3} \mathrm{Ge}$. In the latter case the $\mathrm{DCO}$ was characterized by a jump in $F_{p}$, whereas in our samples the transi-

TABLE I. $L_{c} / d$ for several temperatures in both samples at the crossover field $b_{\text {co }}$ computed with Eq. (5).

\begin{tabular}{cccccc}
\hline \hline Sample & $d(\mu \mathrm{m})$ & $t$ & $b_{\mathrm{co}}$ & $\frac{R_{c}\left(b_{\mathrm{co}}\right)}{a_{0}}$ & $L_{c, \mathrm{co}} / d$ \\
\hline \multirow{3}{*}{ I } & \multirow{2}{*}{2.0} & 0.248 & 0.82 & 20 & $0.63( \pm 0.10)$ \\
& & 0.579 & 0.83 & 34 & $1.39( \pm 0.26)$ \\
& & 0.827 & $\cdots$ & $\ldots$ & $\ldots$ \\
& & & & \\
II & \multirow{4}{*}{15.5} & 0.245 & 0.80 & 100 & $0.50( \pm 0.08)$ \\
& & 0.408 & 0.82 & 101 & $0.52( \pm 0.09)$ \\
& & 0.680 & 0.75 & 132 & $0.87( \pm 0.23)$ \\
& & 0.816 & $\cdots$ & $\cdots$ & $\cdots$ \\
\hline \hline
\end{tabular}


tion is rather continuous. In addition, the decay of $F_{p}$ above the maximum in Fig. 1 is much slower than previously observed. ${ }^{9}$ We think this less distinct behavior, like the large $\Delta T_{c}$ and $\Delta B$ obtained above, is caused by the inhomogeneities in our single crystals which will smear out all the sharp features and there is no contradiction with our conclusion that the peak effect reveals a DCO.

In summary we have measured the perpendicular field dependence of $F_{p}$ for several temperatures in very thin single crystals of $2 H-\mathrm{NbSe}_{2}$. Below $b_{\text {co }} F_{p}$ is well described by the 2DCP theory for an elastically deformed FLL. It is argued that at $b_{\text {co }}$ screw dislocations enter the flux-line lattice and destroy the positional correlation along the field direction, thereby changing the disorder in the FLL from 2D to 3D. It is argued that the correlation perpendicular to the field is maintained at $b_{\text {co }}$, and that the peak in $F_{p}$ is mainly caused by a decrease of $L_{c}$. At $b_{\text {co }}$ the crossover criterion $L_{c}=d / 2$ is fulfilled with $L_{c}$ given by $\mathrm{Eq}$. (5), in which for the first time both the electron-mass anisotropy related to the layered structure of our crystal and the dispersion of $c_{44}$ have been taken into account.

We would like to thank Dr. G. A. Wiegers for providing us with single crystals and Professor J. A. Mydosh for his stimulation and interest. This work is part of the research program of the Foundation for the Fundamental Research on Matter.
'A. Houghton, R. A. Pelcovits, and A. Subdø, Phys. Rev. B 40, 6763 (1989).

${ }^{2}$ P. H. Kes, J. Aarts, V. M. Vinokur, and C. J. v.d. Beek, Phys. Rev. Lett. 64, 1063 (1990).

${ }^{3}$ D. R. Nelson, Phys. Rev. Lett. 60, 1973 (1988).

${ }^{4}$ E. H. Brandt, Phys. Rev. Lett. 63, 1106 (1989).

${ }^{5}$ Y. Yeshurun and A. P. Malozemof, Phys. Rev. Lett. 60, 2202 (1988).

${ }^{6}$ M. V. Feigel'man and V. M. Vinokur, Phys. Rev. B 41, 8986 (1990).

${ }^{7}$ M. P. A. Fisher, Phys. Rev. Lett. 62, 1415 (1989).

${ }^{8}$ R. H. Koch, V. Foglietti, W. J. Gallagher, G. Koren, A. Gupta, and M. P. A. Fisher, Phys. Rev. Lett. 63, 1511 (1989).

${ }^{9}$ R. Wördenweber and P. H. Kes, Physics 135B, 136 (1985); R. Wördenweber and P. H. Kes, Phys. Rev. B 34, 494 (1986); P. H. Kes and R. Wördenweber, J. Low Temp. Phys. 67, 1 (1987); R. Wördenweber and P. H. Kes, Cryogenics 29, 321 (1989).

${ }^{10}$ E. H. Brandt, J. Low Temp. Phys. 64, 375 (1986); E. H. Brandt and U. Essman, Phys. Status. Solidi B 144, 13 (1987); H. R. Kerchner, J. Low Temp. Phys. 50, 337 (1983).

"A. I. Larkin and Yu. N. Ovchinnikov, J. Low Temp. Phys. 34, 409 (1976).

${ }^{12}$ P. H. Kes and C. C. Tsuei, Phys. Rev. Lett. 47, 1930 (1981); Phys. Rev. B 28, 5126 (1983).
${ }^{13}$ B. E. Brown and D. Y. Beernsten, Acta Crystallogr. 18, 31 (1965).

${ }^{14}$ N. Toyota, H. Nakatsuji, K. Noto, A. Hoshi, N. Kobayashi, Y. Muto, and Y. Onodera, J. Low Temp. Phys. 25, 485 (1976).

${ }^{15}$ P. de Trey, S. Gygax, and J. P. Jan, J. Low Temp. Phys. 11, 421 (1973).

${ }^{16}$ E. H. Brandt, J. Low Temp. Phys. 26, 709 (1977); 26, 735 (1977); 28, 263 (1977); 28, 291 (1977).

${ }^{17}$ P. H. Kes and J. v.d. Berg, in Studies of High Temperature Superconductors, edited by A. V. Narlikar (NOVA, New York, 1990), Vol. 5, p. 83.

${ }^{18}$ H. F. Hess, R. B. Robinson, R. C. Dynes, J. M. Valles, Jr., and J. V. Waszczak, Phys. Rev. Lett. 62, 214 (1989).

${ }^{19}$ P. H. Kes and C. C. Tsuei, J. Non-Cryst. Solids 61-62, 865 (1984).

${ }^{20}$ R. Wördenweber, A Pruymboom, and P. H. Kes, J. Low Temp. Phys. 70, 253 (1988); A. Pruymboom, Ph.D. thesis, Leiden University, 1988 (unpublished).

${ }^{21}$ C. S. Pande, Appl. Phys. Lett. 28, 462 (1976).

${ }^{22}$ E. J. Kramer, Philos. Mag. 33, 331 (1976).

${ }^{23}$ E. H. Brandt, Phys. Rev. Lett. 57, 1347 (1986).

${ }^{24}$ R. Wördenweber, P. H. Kes, and C. C. Tsuei, Phys. Rev. B 33, 3172 (1986). 